\title{
Supply and Demand Oriented Energy Management in the Internet of Things
}

\author{
Qingjuan Li1 ${ }^{1}$ Hong Liu², Huansheng Ning1, Yang Fu³ , Songde $\mathrm{Hu}^{1}$, Shunkun Yang ${ }^{4}$ \\ ${ }^{1}$ School of Computer and Communication Engineering, University of Science and Technology Beijing, Beijing, \\ China \\ ${ }^{2}$ Engineering Research Institute, Run Technologies Co., Ltd. Beijing, Beijing, China \\ ${ }^{3}$ Shenyang Branch, Texas Instruments Semiconductor Technologies (Shanghai) Co., Ltd., Shanghai, China \\ ${ }^{4}$ School of Reliability and Systems Engineering, Beihang University, Beijing, China \\ Email: ustbliqingjuan@sina.com
}

Received 9 January 2016; accepted 28 January 2016; published 31 January 2016

Copyright (C 2016 by authors and Scientific Research Publishing Inc.

This work is licensed under the Creative Commons Attribution International License (CC BY).

http://creativecommons.org/licenses/by/4.0/

\section{(c) (i) Open Access}

\begin{abstract}
The Internet of Things (IoT) is emerging as an attractive paradigm involving physical perceptions, cyber interactions, social correlations and even cognitive thinking through a cyber-physical-social-thinking hyperspace. In this context, energy management with the purposes of energy saving and high efficiency is a challenging issue. In this work, a taxonomy model is established in reference to the IoT layers (i.e., sensor-actuator layer, network layer, and application layer), and IoT energy management is addressed from the perspectives of supply and demand to achieve green perception, communication, and computing. A smart home scenario is presented as a case study involving the main enabling technologies with supply-side, demand-side, and supply-demand balance considerations, and open issues in the field of IoT energy management are also discussed.
\end{abstract}

\section{Keywords}

Internet of Things, Internet of Energy, Energy Management, Smart Home, Communication, Green Computing

\section{Introduction}

The Internet of Things (IoT) is endowed with implications regarding physical perception, cyber interaction, social correlation and cognitive thinking for the establishment of a ubiquitous and intelligent cyber-physical-social thinking hyperspace. The future IoT is expected to provide reliable communication, intelligent computing, and high efficiency characterized by interconnection, intelligence, and greenness [1]. Because of limited com- 
munication, networking and infrastructure resources, energy management strategies are important for the sustainable development of the IoT and will have the potential to significantly reduce energy consumption and enhance energy efficiency.

Energy management is a long-standing issue, and energy management solutions have been designed to achieve long network lifetimes by reducing and balancing the energy consumption in heterogeneous networks. In the context of information and communications technology (ICT), energy management approaches are primarily focused on reducing energy demands through the use of green communication and data centers. Unlike the energy management in traditional networks, IoT energy management faces serious challenges as a result of the IoT features of ubiquitous sensing, networking, and computing. Thus, additional considerations must be addressed for IoT energy management.

In the IoT, energy supply and demand will become unprecedentedly complex as a result of global greenhouse gas (GHG) emissions. For instance, it is estimated that the network energy consumption in Japan in 2025 will be 13 times compared to the 2006 level and equal to $10 \%$ of Japan's total power output. With the ubiquitous connection of things to the Internet, energy consumption will climb an increasing rate. When considering sustainable energy strategies, IoT energy management is thus becoming a noteworthy research topic, involving complex issues of energy supply, energy demand, and supply-demand balance. To trace the evolution of energy management, the development of the relevant strategies can be divided into four stages: the initial energy management stage, the advanced energy management stage, the green ICT stage, and the green IoT stage.

- Initial energy management stage: the communication technologies of this stage are predominantly focused on device design and testing. Because of restrictions related to the device manufacturing process, energy management is not a primary design target. Instead, the main target of communication and networking technologies is to improve system performance. Energy management may be not a prominent concern in smallscale networks during the initial energy management stage.

- Advanced energy management stage: the second stage is characterized by wide-range deployment and coverage of networks and communication infrastructures. In the advanced energy management stage, the primary purpose of energy management is to ensure an uninterrupted supply of power to the communication and networking infrastructures.

- Green ICT stage: the third stage begins with a rapid increase in data traffic due to user-centric applications. As a result of increasing energy consumption and GHG emissions from Internet infrastructures, energy management strategies are primarily focused on reducing the energy consumption of network terminal devices.

- Green IoT stage: the fourth stage develops alongside the IoT and accelerates the evolution of the Internet of Energy (IoE). Green communications, networking and information technologies are applied to achieve energy conservation and emission reduction. The energy flow and information flow are traced throughout the power cycle, including generation, transmission, distribution, and utilization.

With the evolution of the IoT into the green IoT stage, energy management efforts face severe challenges. In this work, we focus on supply- and demand-oriented energy management in the IoT context, and the main contributions of the study are as follows.

1) A taxonomy model is established for IoT energy management, in which the IoT layers (i.e., the sensor-actuator layer, network layer, and application layer) and the energy aspects (i.e., supply-side, demand-side, and supply-demand balance concerns) are jointly considered with regard to energy management to achieve green perception, green communication, and green computing.

2) A smart home scenario is introduced as a case study of energy management, in which the main enabling technologies, including photovoltaic power generation, the micro-grid concept, virtualization, software-defined networking, advanced metering infrastructure, demand response, and vehicle-to-grid technology, are each discussed with regard to achieving high efficiency and energy conservation.

3) New perspectives on IoT energy management are discussed, involving aspects of cyber-physical space fusion, the convergence of communication and computing, and the IoE.

The remainder of the paper is organized as follows. Section 2 presents the proposed taxonomy model of IoT energy management in the sensor-actuator layer, network layer, and application layer. Section 3 considers a smart home scenario as a case study to introduce enabling technologies based on supply-side, demand-side, and supply-demand balance considerations. Section 4 discusses new perspectives on IoT energy management. Finally, Section 5 presents the conclusion. 


\section{The Taxonomy Model of Energy Management in the IoT}

In the IoT context, the smart grid concept is becoming the next-generation power grid technology that will transform the traditional power grid into a new energy paradigm and achieve more environmentally friendly electrical energy management to increase the connectivity, automation and coordination among energy suppliers and consumers. Figure 1 depicts the proposed taxonomy model of energy management in the IoT, which illustrates the classification of energy management in the IoT. In the taxonomy model, a two-dimensional mapping is established based on the IoT layers and their corresponding energy aspects. In one dimension, the energy management issues related to the sensor-actuator layer, network layer, and application layer are presented. In the other dimension, energy management issues are analyzed based on considerations related to the supply side, demand side, and supply-demand balance. The main objectives of green perception, green communication, and green computing are sequentially the focuses of the individual layers from bottom to top. These two dimensions reconstruct the issues related to IoT energy management into an interrelated mapping framework, based on which both the energy interactions and information interactions involved in IoT energy management can be understood.

\subsection{Sensor-Actuator Layer}

The sensor-actuator layer converts physical objects into cyber entities and comprises the generalized sensors used to perform target identification and the cyberization of physical objects. The sensing techniques employed in this layer mainly include ZigBee, radio frequency identification (RFID), Wi-Fi, Bluetooth, infrared induction, the Global Positioning System (GPS), and radar. Note that mechanical and electronic actuators (e.g., valves and switches) may be connected to the sensors to execute issued instructions based on the sensed data. The sensoractuator layer performs the tasks of energy harvesting and storage, efficient sensing and processing, and energy buffering, corresponding to the supply side, the demand side, and supply-demand balance, respectively.

\subsubsection{Energy Harvesting and Storage}

In the sensor-actuator layer, the supply-side energy management strategy should address the heterogeneity arising from the ubiquitous collection of resources, and thus, distributed and hybrid energy harvesting and storage become the predominant energy supply trends. Energy harvesting refers to capturing minute amounts of energy that would otherwise be lost in the forms of heat, light, sound, vibration, electromagnetic waves, and others. This energy is obtained from one or multiple naturally or artificially occurring power sources, and the collected energy can be accumulated, stored, and managed for later use. The energy interactions related to energy harvesting primarily involve the diverse power sources from which energy is collected.

1) Renewable energy: renewable energy is an important source of power for unattended sensors and actuators, and it is generally managed by ultra-low-power management circuits for sustaining sensing and actuating operation, in which sensor nodes can be implemented to sense the current meteorological conditions by indirectly measuring the equivalent electrical parameters. Wind and solar are the most prominent renewable energy sources, accounting for $62 \%$ and $13 \%$, respectively, of the total non-hydro renewable electricity provision capacity.

a) Solar energy: solar energy is an inexhaustible power source that can be transformed into heat or electricity via the photovoltaic effect. The exploitation of solar energy is particularly suitable for wireless, selfsufficient, portable electronic devices. Considering the relatively high power density of outdoor applications, solar energy

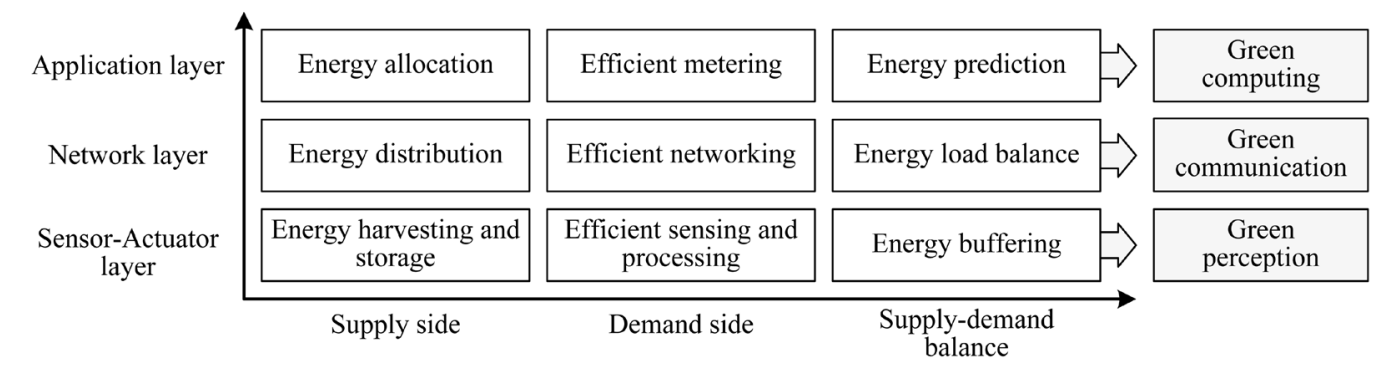

Figure 1. The taxonomy model of energy management in the IoT. 
is the most readily available source for resource-constrained systems. Semiconducting polymer solar cells are attractive devices for low-cost solar energy harvesting based on composite materials with a bulk heterojunction structure. However, polymer solar cells have low power conversion efficiency and are potentially unstable under exposure to light, and they cannot be applied in harsh environments. Alternatively, nanotechnology can be beneficial for photovoltaic and photoelectrochemical applications in solar energy harvesting, and nanomaterials can potentially provide enhanced approaches to enabling generation III solar technologies. Solar photovoltaic generation can be formalized in terms of current-voltage characteristic equations, which are related to the solar cell parameters and environmental conditions (e.g., radiant emittance, irradiance conditions, and temperature).

b) Wind energy: devices for wind energy conversion can be classified into variable-speed devices and fixedspeed devices. The former employ indirect electronic interfaces for connection to the grid, and the latter rely on squirrel-cage induction generators for direct grid connection. In wireless sensor networks (WSNs), wind energy can be provided through ultra-low-power management circuits for sustained operation, in which wind-driven sensor nodes can be implemented to sense meteorological conditions by indirectly measuring the equivalent electrical parameters. Wind turbine power generation can be modeled as a function of the wind speed, and timeseries analyses of power and wind speed data are useful for wind energy prediction.

2) Radio frequency (RF) energy: RF energy is a prevalent source of power for electromagnetic devices. Radio signals are persistently transmitted into the surrounding environment to enable the wireless charging of low power devices with high mobility, usability and reliability. Typically, RF energy is broadcast in specific frequency bands (e.g., $13.56 \mathrm{MHz}, 915 \mathrm{MHz}, 2.4 \mathrm{GHz}$, and $5.8 \mathrm{GHz}$ ), and this RF energy supports both batterybased and battery-free devices. Of these, the battery-based devices are charged to eliminate the need for disposable battery replacement, and the battery-free devices are primarily designed for on-demand operation. RFenergy components usually require no additional energy-consuming circuitry for maximum power point tracking (MPPT). Alternatively, a wireless-interface system-on-a-chip (SoC) comprises an RF transmitter and a microcontroller for battery-free short-range transmission.

3) Mechanical energy: mechanical energy is obtained by converting mechanical vibrations into electricity, predominantly through the piezoelectric, electrostatic, and electromagnetic effects. Among these, the piezoelectric effect is used to generate energy via mechanical pressure, the electrostatic effect is activated by relative movement between electrically isolated charged capacitor planes, and the electromagnetic effect arises upon a relative motion between a magnetic flux and a conductor. Such harvesting approaches are increasingly emerging for the collection of diverse types of mechanical energy. For example, kinetic energy originating from the human body (e.g., heartbeat and body movements) can be sensed and applied in human wireless networks.

4) Thermal energy: thermal energy is obtained through the thermoelectric and pyroelectric effects, in which thermal energy (e.g., temperature gradients and temperature variations) is converted into electric power. Thermoelectric power generators (TEGs) convert unused but available heat from ambient thermal sources into electricity; such heat sources exist in many different forms (e.g., hot surfaces; heating, ventilation and air conditioning (HVAC) systems; and the human body). In particular, the pyroelectric effect offers an approach to powering distributed sensor and actuator networks for remote energy support and maintenance.

Energy storage is accomplished by using physical media devices (e.g., accumulators) to store the acquired energy for direct or indirect use. Additionally, energy storage systems are emerging for power peak shaving and renewable energy integration in smart power grid applications. The energy interactions related to energy storage predominantly involve electrical and thermal energy storage technologies.

1) Electrical energy storage: electrical energy storage directly or indirectly provides electrical energy via external electrical interfaces. In the IoT, various electrical approaches become prevalent, including chemical energy storage (e.g., bio-fuels, liquid nitrogen, hydrogen, hydrogen peroxide, and photocatalytic storage), electrochemical energy storage (e.g., batteries and flow cells), mechanical energy storage (e.g., flywheels, compressed air, wind, hydraulic accumulators, and micro-mechanical devices), and electromagnetic energy storage (e.g., capacitors, supercapacitors, and superconducting magnetic energy storage (SMES)).

2) Thermal energy storage: thermal energy storage technologies can exploit the sensible and latent heat capacities water heating, waste heat utilization, cooling and air conditioning. These technologies mainly include systems based on cryogenic liquid air or nitrogen, ice storage, eutectic systems, fireless locomotives, molten salts, seasonal thermal stores, steam accumulators, and other custom thermal storage media. Phase change materials (PCMs) have become an attractive basis for the development of thermal energy storage technologies because of their high energy storage density and isothermal behavior during phase change processes. 
In the IoT, energy storage relies on hybrid approaches for different practical considerations. Among these, electrochemical and electromagnetic approaches are popular in hybrid electric vehicle applications, in which capacitors are directly switched across a direct current link to the motor drive during peaks in power demand. Meanwhile, mechanical and electromagnetic approaches can also be integrated into overall energy systems. For instance, a superconducting flywheel energy storage unit can store rotational mass as kinetic energy and then release it upon demand. Moreover, hybrid energy storage approaches have certain obvious advantages, including high efficiency, long life cycles, and a wide range of operating temperatures.

Supercapacitors have also emerged as storage components instead of rechargeable batteries. Supercapacitors as a typical electromagnetic approach are most suitable for buffering unstable environmental energy. Supercapacitors exhibit a high charge-discharge efficiency (e.g., 97\% - 98\%) and an infinite life cycle, and they have come to serve as a foundation for alternative energy buffer approaches in frequent pulsing applications. However, supercapacitors self-discharge at a higher rate than do rechargeable batteries.

\subsubsection{Efficient Sensing and Processing}

Demand-side energy management is primarily focused on reducing energy consumption, enhancing energy conservation, achieving energy minimization, and improving energy efficiency. Efficient sensing and processing depends on the materials, operating principles, and manufacturing processes of the applied sensors. Next-generation ultra-low-power sensors are important for reducing energy consumption in the sensor-actuator layer. For instance, in video monitoring scenarios, the energy consumed by video cameras represents the largest contribution to the total energy consumption. Dynamic energy processing based on lower-power circuits, for which dynamic voltage and frequency scaling (DVFS) and dynamic power management (DPM) are typical approaches, is a promising means of reducing energy consumption.

1) DVFS is an approach to real-time power management in which a microprocessor's clock frequency is decreased to allow a corresponding reduction in the voltage supply. Traditional DVFS transition overhead schemes suffer from significant inaccuracies (e.g., inaccuracies in the DC-DC converters and frequency synthesizers). Incorrect and inaccurate DVFS transition overheads create obstacles to determining the precise break-even time and cause unnecessary energy losses. Additionally, dynamic voltage scaling (DVS) is also an efficient method of dynamically adjusting the system supply voltage to reduce energy consumption. A DC-DC converter is a key electronic device for voltage regulation in very-large-scale integration (VLSI) and should be designed to minimize the total dissipated energy and peaks therein. In practical applications, multicore processors are popular for decreasing the voltage and clock frequency to save energy at the cost of increased delay [2]. Optimal clock frequencies can be designed to minimize energy use in both local and global DVFS with scheduling considerations, in which the trade-off between the selection of clock frequencies and scheduling is a key issue for minimizing energy consumption.

2) DPM refers to the dynamic reconfiguration of an electronic system to provide the requested services while minimizing the loads of the system components, in which energy conservation is achieved by selectively shutting down or reducing the performance of idle components. A hierarchical DPM strategy can be designed to enhance the energy efficiency of system components (e.g., microprocessors and interfaces) in an embedded system. Field programmable gate arrays (FPGAs) are available in multiprocessor systems on chips (MPSoCs) to reduce energy consumption based on the idle times of processors. To formulate the rules governing the switch from a high energy-consuming state to a low-energy-consuming state, Markov decision processes (MDPs) with statedependent control are suitable for several types of applications. However, MDPs become unfeasible in the case of a state dependent policy requiring that all states in a request arrival process must be observed during online monitoring [3], and an optimal time-out policy should be designed to minimize the expected power consumption per unit time in the steady state.

\subsubsection{Energy Buffering}

In the sensor-actuator layer, energy buffering can be regarded as a technology for turning local power on and off to cooperatively achieve a global energy balance. Rechargeable batteries are the most common means of buffering unstable energy from ambient environmental energy sources. The most popular rechargeable technologies include sealed lead acid (SLA), nickel cadmium (NiCd), nickel metal hydride (NiMH) and lithium-ion batteries. Rechargeable batteries have a limited lifetime; they suffer performance attenuation after a certain number of cycles of charging and discharging operations. However, in IoT energy management, an energy buffer must be 
charged whenever its energy source is available and must also be capable of on-demand discharge, meaning that most rechargeable batteries are unsuitable for IoT energy buffering in dynamic environments.

Regarding the reduction of electricity costs and the improvement of energy efficiency in IoT applications, energy buffering faces several challenges. Considering the inherent heterogeneity of the physical characteristics of energy storage devices (e.g., their charging and discharging rates), receding horizon control (RHC)-based multi-tier energy buffering management could be a suitable strategy for optimizing renewable energy utilization [4]. For residential hybrid electrical energy storage systems, energy buffering strategies decrease users' electric bills by performing charging operations during low price periods and releasing stored energy during high-price periods. Such energy buffering strategies based on deferrable operations achieve the intelligent dispatch of distributed energy storage with long-term benefit maximization. The optimal decision problem of energy buffering can be formulated as a discounted-cost MDP over an infinite horizon [5] [6]. In particular, in a system with multiple sensor nodes and a single energy-harvesting source, energy buffering can be used to store the energy harvested from ambient energy sources and to share energy among the sensor nodes such that the long-run average delay during data transmission is minimized.

\subsection{Network Layer}

The network layer includes multiple network components (e.g., interfaces, routers, and gateways) and communication channels. Heterogeneous network configurations are established based on WSNs, the Internet, mobile communication networks, telecommunication networks, and other network technologies. This layer ensures reliable data transmission by applying secure data coding, fusion, mining, and aggregation algorithms, and it realizes interconnections among heterogeneous networks. The network layer performs the tasks of energy distribution, efficient networking, and energy load balancing, corresponding to the supply side, the demand side, and supply-demand balance, respectively.

\subsubsection{Energy Distribution}

Energy distribution is expected to be more efficient in hybrid energy systems, in which the penetration of combined heat-and-power-based distributed energy generation has resulted in interconnections among the electricity, water, and natural gas distribution networks. In an energy context with bi-directional power and information flows, optimal real-time energy distribution can be achieved using distributed online algorithms that consider factors such as power grid load smoothing, dynamic price strategies, and energy provisioning costs [7]-[9].

Renewable energy resources must be incorporated into an existing energy distribution system, and increasing attention is being directed toward maximizing energy efficiency in this attempt. For instance, the need for reliable energy distribution may motivate the implementation of photovoltaic energy systems that monitor environmental variables and provide flexible management of solar power. Because the integration of distributed energy resources (DERs) can potentially impact the energy distribution operations by affecting equipment reliability and power quality [10], convex optimization can be introduced into energy distribution schemes to exploit energy price arbitrage. In the case that megawatt-scale wind turbines are connected into an existing distribution system, the voltage stability of the distribution feeders will be obviously influenced. In such a scenario, distributed wind turbine generators (WTGs) with appropriate controllers could enhance the stability of the grid [11], and synchrophasor-based auxiliary controllers based on distribution phasor measurement units are useful for stabilizing the voltage deviations of energy distribution feeders. Meanwhile, the small-signal stability performance is also a challenging issue for renewable-energy based distribution systems in which synchronous, induction, and static generators supply energy for static and dynamic loads.

\subsubsection{Efficient Networking}

Demand-side energy management for green communication aims at reducing energy consumption in the network layer. In the IoT, the network layer includes multiple network components (e.g., interfaces, routers, and gateways) and communication channels. Note that the included management and data centers act as network nodes for communication. Heterogeneous network configurations may be established based on WSNs, the Internet, mobile communication networks, and telecommunication networks. It has been indicated that the energy consumed by access networks, including wired access networks and wireless access networks, accounts for $70 \%$ of the total energy consumption, whereas transport and core networks account for $30 \%$. Thus, strategies for demand-side energy management are predominantly focused on the following types of networks. 
1) Wired access networks: wired access networks represent a large percentage of the energy consumption in the network layer, encompassing the energy consumptions from digital subscriber lines, hybrid fiber-coaxial networks, passive optical networks, fiber-to-the-node networks, point-to-point optical systems, universal mobile telecommunications systems, and WiMAX. Fiber communication technologies offer high capacity, meaning that fewer cables are needed and less energy loss is incurred. Meanwhile, optical signals can travel long distances without requiring amplifiers, which are some of the most energy-demanding components in such networks. Moreover, non-IT equipment accounts for nearly half of the total energy consumption of wired access networks, and technology that provides efficient, uninterrupted power supply and cooling capabilities can be used for energy conservation.

2) Wireless access networks: the IoT merges fixed, mobile, and wireless communications to establish heterogeneous communications. Radio base stations (RBSs) are the most energy-greedy components of wireless access networks; their modes of energy dissipation include power supply system losses, climate control systems, batteries, backend interfaces, and transceivers. To reduce the energy consumption of RBSs, energy-efficient solutions have been proposed both at the device level (e.g., energy efficient amplifiers and energy-efficient antennas) and at the network level (e.g., fem to cell networks, relaying, and RBS shut-off).

3) Backbone networks: the main energy-demanding components of backbone networks are high-end router and packet switching devices, and a reduction in energy demand can be achieved through re-engineering, dynamic adaption, and sleep/standby approaches. Optical packet switching (OPS) is a promising means of improving energy efficiency and capacity; electronic packet switching (EPS) and hybrid packet switching (HPS) are two specific types of OPS systems. OPS routers apply all-optical contention resolution strategies without relying on store and forward methods to achieve high energy efficiency.

\subsubsection{Energy Load Balancing}

In the network layer, the goal of supply-demand balance is to achieve load balancing during communication and networking. Cluster heads are usually subject to heavy traffic loads for gathering and relaying information, and they may deplete energy resources, resulting in service disruptions in WSNs. With the penetration of distributed energy generation (e.g., renewable energy sources), network operators are facing new challenges in ensuring energy balance and the quality of energy service, and uncontrolled energy generation may have a negative impact on such efforts. To balance the traffic loads and energy consumption in WSNs, the role of the cluster head should be rotated among all available nodes, and the cluster sizes should be determined based on the network capacity. Energy-efficient clustering algorithms are popular means of determining suitable cluster sizes based on the hop distance to the data sink to achieve approximate equalization of node lifetimes [12], and maximum connected load balancing algorithms can be designed to achieve full coverage and connectivity preservation by dynamically establishing load-balanced routing cover trees. Typical routing protocols, including traffic-size-aware routing, dynamic load-aware routing, load-balanced ad hoc routing, receiver oriented load-balancing reliable routing, load-balancing multipath routing, and ring-based energy-aware routing, can be applied in mobile ad hoc networks (MANET) to divert traffic from congested routes.

With the proliferation of cloud ecosystems, Internet data centers (IDCs) are facing severe energy pressures, and unified spatio-temporal load balancing can be applied to optimize energy costs in distributed IDCs [13]. Geographical load balancing has become a noteworthy approach for reducing electricity costs for service providers by exploiting the differences in electricity prices across regions. However, such a reduction in electricity cost may lead to an increase in total energy consumption. Moreover, queuing and transmission delays affect the quality of service for real-time interactive systems, in which the transmission delay problem can be formulated as a mixed-integer nonlinear programming (MINLP) problem to achieve optimal load balancing and energy cost management for IDCs [14] [15]. Meanwhile, idle and light-loaded servers can be switched into a sleep state to save energy.

\subsection{Application Layer}

The application layer provides services to support practical IoT scenarios involving different area networks, of which energy allocation, efficient metering, and energy prediction correspond to the supply side, the demand side, and supply demand balance, respectively. 


\subsubsection{Energy Allocation}

Energy allocation can be formulated as an infinite-horizon MDP to achieve throughput maximization. In rechargeable WSNs, the sensors are subject to time-varying energy harvesting rates, battery availability, data buffer availability, and channel fading, thereby presenting challenges in energy allocation for energy harvesting applications. A methodology for optimal transmission energy allocation has been proposed for the remote state estimation of linear stochastic dynamical systems [16], in which optimal energy allocation is applied for Kalman filtering over packet dropping links with imperfect acknowledgments and energy harvesting constraints. In heterogeneous networks powered by hybrid energy sources, energy optimization requires both user association optimization in the spatial dimension and energy allocation in the time dimension [17]. This two-dimensional energy optimization addresses two independent issues: the user association optimization is a convex optimization problem with the goal of minimizing the total energy consumption by balancing the traffic across different base stations within a certain time slot, whereas the energy allocation is performed for each individual base station across different time slots to lexicographically minimize the energy consumption.

In an energy harvesting transmitter based on a hybrid energy storage model (e.g., a supercapacitor and a battery), the supercapacitor has a finite storage capacity, whereas the battery has an unlimited capacity. The transmitter determines whether to store the harvested energy in the supercapacitor or the battery; for this purpose, optimal energy allocation strategies should be considered. Real-time DER allocation has become the main focus of IoT applications, along with the utilization of hybrid energy sources. Intelligent algorithms such as the minority game algorithm [18], binary quantum-behaved particle swarm optimization, iterative algorithms, and low-complexity optimal algorithms should be designed to achieve energy-efficient resource allocation. Nonlinear fractional programming can be applied to formulate an energy optimization problem for energy-per-bit minimization.

\subsubsection{Efficient Metering}

Smart meters are expected to support bi-directional information interactions between power providers and residential customers to enable real-time energy information collection. This is necessary to achieve the efficient energy metering and remote monitoring of energy nodes, to which end a smart meter is an advanced device for recording the consumption of electrical energy and other electrical parameters for automatic meter reading. Such a meter possesses a central control unit, which includes a neuron chip and an energy metering chip. The neuron chip is programmed to calculate the energy parameters for tracking the charging and discharging of power, whereas the purpose of the energy metering chip is to obtain the necessary values to perform appropriate scaling operations. The optical voltage transducer (OVT) and optical current transducer (OCT) are two key components in the design of energy metering systems, which is subject to several standards and protocols, including the Device Language Messaging Specification/Companion Specification for Energy Metering (DLMS/COSEM) and the Internet based Session Initiation Protocol (SIP) signaling protocol in the application layer [19]. The challenging issues include the gathering of real-time data transmissions, the various communication modes (e.g., uni-casting, multi-casting, broadcasting, and geo-casting), and secure wireless interactions.

Google launched the Power Meter project to assist consumers in tracking their electricity usage, with a focus on renewable energy, electricity grid upgrades, and other measures for reducing GHG emissions. Microsoft also built an online web application, Hohm, to allow consumers to analyze their energy usage and provide energysaving recommendations. However, these high-profile attempts have had little effect because of their insufficient scope and a lack of data at adequate bandwidths [20]. Generally, energy meters provide adequate data with high performance and at a reasonable hardware cost; thus, efficient signal processing algorithms should be designed to ensure high-bandwidth measurements of voltage, current, harmonics, and power.

Additionally, energy theft detection has become a noteworthy concern in the implementation of efficient metering in home area networks, neighborhood area networks, and wide area networks. A tree-based threat model has been proposed to illustrate the energy theft behaviors relevant to energy meters [21], in which six types of attackers (i.e., curious eavesdroppers, greedy customers, malicious eavesdroppers, swanky attackers, active attackers, and intrusive data management agencies) are defined. There are two main classes of energy theft detection techniques: classification-based detection and state-estimation-based detection.

1) Classification-based detection applies machine learning and artificial intelligence algorithms (e.g., support vector machines (SVMs), fuzzy classification, and neural networks) to obtain a suitable classifier based on sample data that are frequently collected by meters. 
2) State estimation based detection adopts additional monitoring mechanisms (e.g., WSNs and RFID) to improve the detection rate and reduce the incidence of false positives. This detection schemes offers high accuracy based on precise parameter estimation but has costly hardware requirements.

In addition, game-theory-based detection has emerged as a new perspective from which to address energy theft issues by formulating the problem as a game between the electric utility and the electricity thief. A reasonable solution can be established to reduce electricity losses under the assumptions that a thief will steal a predefined amount of electricity while minimizing the likelihood being detected and that a utility institution will strive to maximize the probability of detection. Heterogeneous data fusion should be considered to combine sensor data with the consumption data from a smart meter to more accurately detect energy theft.

\subsubsection{Efficient Metering}

Energy prediction offers two functionalities that are beneficial for supply-demand balancing: one is to predict the future energy situation within an appropriate time period, and the other is to schedule tasks or adjust the operational state in response to current energy trends. Considering the dynamic reconfiguration of wireless communication networks, more energy and time are required to generate reasonable predictions for applications involving reconfigurable systems. To address this issue, estimated weighted moving average (EWMA) and weather-conditioned moving average (WCMA) prediction algorithms can be applied in energy prediction schedulers [22]. Supervisory-control-strategy-based energy demand prediction is helpful for minimizing energy consumption in real-time operations. For instance, for applications related to plug-in hybrid electric vehicles, energy demand prediction is performed via three successive steps [23]: a neural network model is used to predict a vehicle's energy demand, a mathematical model is used to translate the predicted energy demand into a state of charge (SOC), and an adaptive equivalent consumption minimization strategy is used to track the SOC for power-state determination.

In the IoT, prediction-aware energy-saving algorithms are receiving increasing attention for embedded intelligent terminals (EITs). There are four main types of such energy-saving algorithms, namely, algorithms for the efficient scheduling of tasks, power modeling, the adjustment of working voltages or frequencies, and the reasonable control of transitions among different states [24]. The idle-time-prediction (IP) algorithm, the weighted IP algorithm, the Bayesian IP algorithm, and the running time fixed threshold in IP algorithm are typical energysaving algorithms for controlling transitions among different power consumption states. Moreover, distributed renewable energy facilitates cloud-assisted energy management systems, and renewable energy prediction models are important for predicting the available energy during the scheduling of workloads to match the energy supply [25]. For instance, solar energy prediction is usually achieved based on EWMA and WCMA prediction algorithms, whereas solar energy generation can be modeled as a function that is inversely related to the amount of cloud coverage.

\section{Case Study: Smart Home}

In this section, a smart home scenario is presented as a case study of IoT energy management from the supplyside, demand-side, and supply-demand balance perspectives, as shown in Figure 2. The main enabling technologies, including photovoltaic power generation, the micro-grid concept, virtualization, software-defined networking, advanced metering infrastructure, demand response, and vehicle-to-grid technology, are individually discussed with regard to the attempt to achieve high efficiency and energy conservation.

\subsection{Supply Side}

The issues related to supply-side energy management in a smart home are addressed through the concepts of photovoltaic power generation and micro-grids.

\subsubsection{Photovoltaic Power Generation}

The exploitation of solar energy is particularly suitable for wireless, self-sufficient, portable electronic devices. Considering the relatively high power density of outdoor applications, solar energy is the most readily available source of power for resource-constrained systems. Semiconducting polymer solar cells are attractive devices for low-cost solar energy harvesting based on composite materials with a bulk hetero junction structure. However, polymer solar cells have low power conversion efficiency and are potentially unstable under exposure to light, 


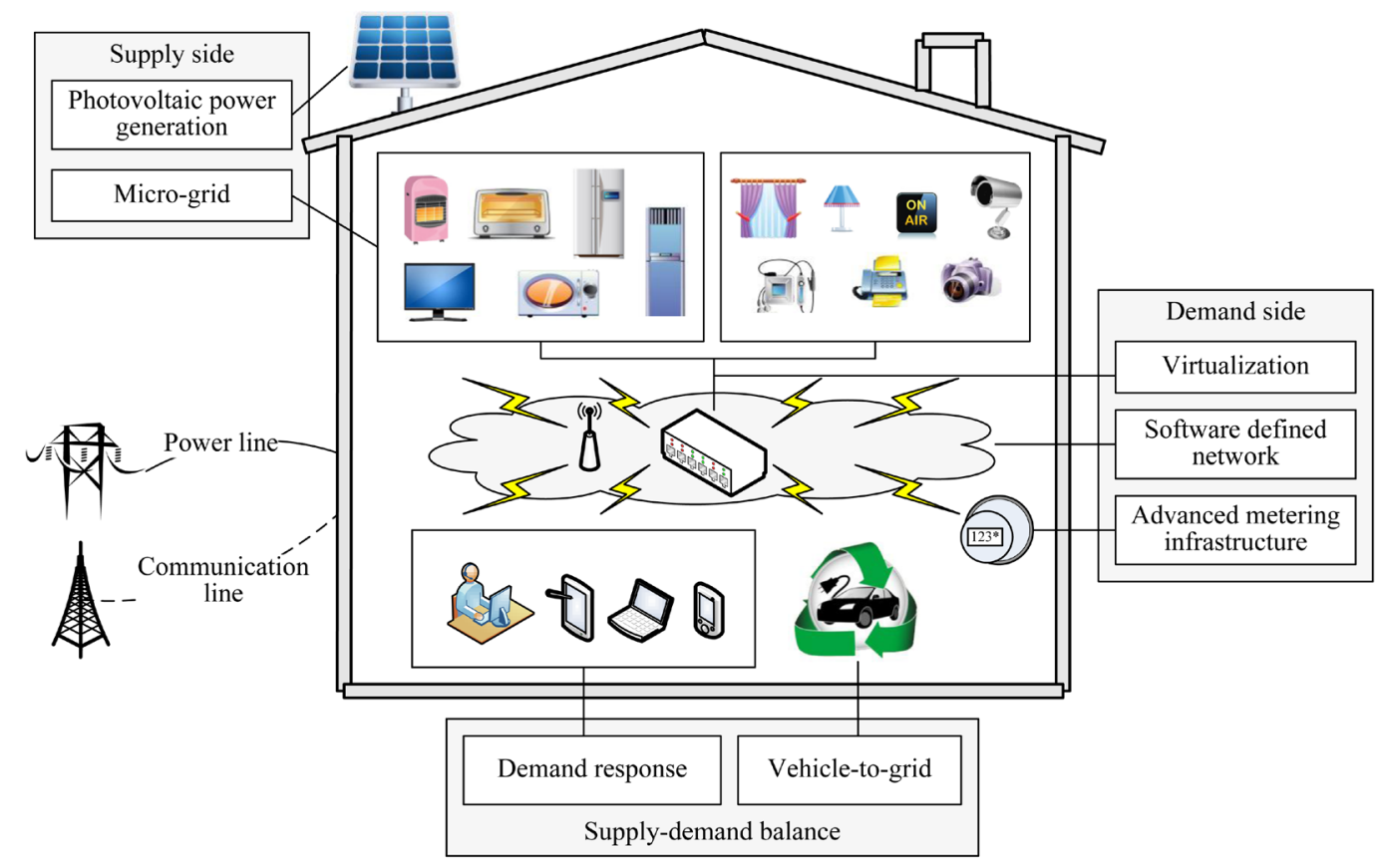

Figure 2. Energy management in a smart home.

and they cannot be applied in harsh environments. Alternatively, nanotechnology can be beneficial for photovoltaic and photoelectrochemical applications in solar energy harvesting, and nanomaterials can potentially provide enhanced approaches to enabling generation III solar technologies.

Photovoltaic power generation technology is predominantly used to supply power for smart household facilities and facilitates the sustainable development of the green IoT. The quasi-Z-source cascade multilevel inverter (qZS-CMI) offers obvious advantages for photovoltaic power systems, including its balanced DC-link voltage and voltage boost capability [26]. However, the qZS-CMI cannot address the intermittent and stochastic fluctuations in the solar power injected into the power grid; for this purpose, a battery for energy storage can be introduced into the qZS-CMI to balance the stochastic fluctuations in photovoltaic power.

To achieve the maximum utilization of solar energy and to ensure steady-state performance and rapid dynamic response, MPPT algorithms can be designed for photovoltaic power generation. However, the power-voltage curve exhibits multiple peaks when partial shading occurs in a photovoltaic power system [27] [28]. Because of the constant-voltage and constant-current characteristics of photovoltaic power, a linear iteration method can be used to estimate the maximum power point with high accuracy. Meanwhile, artificial intelligence techniques (e.g., artificial bee colony algorithms or neurofuzzy inference systems) can provide an efficient solution for delivering the maximum power from a photovoltaic power generation system in standalone operation.

\subsubsection{Micro-Grids}

Micro-grids offer the major advantage of flexibility in operating while either interconnected with the power grid or disconnected in islanded mode, and they predominantly rely on DERs or DER systems for decentralized energy supply. The integration of DERs into the micro-grid architecture enables the establishment of a virtual power plant, providing an incentive for the self-consumption of locally generated power. Such DERs are interfaced with the power grid through electronic power processors, which promote distribution loss reduction and voltage stabilization.

Along with renewable energy penetration, frequency-based power delivery in distributed generators is required for these generators to operate as traditional synchronous generators [29]. However, the frequency is controlled as a common variable throughout the power grid, and it thus a challenge to use frequency-based power sharing to participate in grid frequency control activities. Residential renewable power generation in micro-grids is expected to enable the adjustment of the power level depending on local frequency variations, thereby reducing variations in the power received from the power grid. In a smart home, the operation of smart mi- 
cro-grids can address the challenges encountered in the optimal energy management of the residence with respect to multiple conflicting objectives [30]. Meanwhile, fault-triggered islanding may cause voltage and frequency excursions and induce the disconnection of distributed generation sources, thereby affecting the integrity and availability of the power grid. Typical methods of micro-grid islanding detection include communicationbased, active, and passive methods, and hybrid islanding detection schemes that consider both voltage imbalance and high-frequency impedance are recommended. In a photovoltaic-energy-based micro-grid, the generation of active power may cause the power factor to decrease at the point of common coupling with the power grid. Thus, a photovoltaic coupling voltage source inverter is applied for power control related to active and reactive power operations.

\subsection{Demand Side}

The issues related to demand-side energy management in a smart home are addressed through the concepts of virtualization, software-defined networking, and advanced metering infrastructure.

\subsubsection{Virtualization}

Virtualization refers to the creation of virtual entities representing physical objects for resource reorganization and optimization and can be classified into three main types: full virtualization, partial virtualization, and paravirtualization. Virtual machine (VM) migration has emerged as an essential tool for data center and storage systems for service provisioning and energy-aware consolidation. Network virtualization is an attractive approach to combining available resources into a network by dividing the available bandwidth into independent channels, thereby enhancing energy efficiency. In this context, logical units (e.g., logical switches, logical interfaces, logical routers, logical firewalls, and logical load balancers) can be reproduced in a virtual network topology for unified energy control.

\subsubsection{Software-Defined Networking (SDN)}

Software-defined networking (SDN) is an emerging paradigm for network infrastructures that enables the organization of network services through the abstraction of lower-level functionalities. It is achieved by decoupling the control and data planes; the network intelligence and state are logically centralized, and the underlying network infrastructure is abstracted from the applications [31] [32]. The data plane encompasses the physical network components, including routers, switches, virtual switches, and other wireless access points. The control plane is extracted from the network nodes. The controllers facilitate the addition, update, and deletion of flows and paths during interactions and use the capacity- and demand-related information obtained from the network components through which the traffic flows. Data forwarding at the hardware level is based on the flow and path control information.

Energy communication networks are required to provide energy-efficient and high-security networking, and SDN possesses certain inherent advantages for supporting dynamic network functions and intelligent applications with lower operating costs through the simplification of hardware, software, and management. Recently, the rapid expansion of data centers has aggravated the related energy consumption, which has influenced the sustainable growth of cloud services in the smart home. The SDN technique can be applied for energy aware flow scheduling [33], which means scheduling flows in the time dimension and defining an exclusive routing (EXR) path for each flow. The exclusive occupation of link resources usually results in higher link utilization for each flow, eliminating competition for link bandwidth, implying that the EXR approach achieves higher energy efficiency compared with traditional routing protocols such as regular fair-sharing routing (FSR). SDN differs from network virtualization (NV) and network functions virtualization (NFV), in which virtual tunnels and functions related to physical networks are created. By contrast, in SDN, the physical networks are fundamentally modified to achieve homogeneity of the hardware components by means of a centralized control plane separate from the traffic forwarding or switching plane.

\subsubsection{Advanced Metering Infrastructure (AMI)}

An advanced metering infrastructure (AMI) is an intelligent cyber-physical system that is intended to replace traditional analog devices for automatically measuring, collecting, storing, and analyzing analyzing power information for supply-demand balance. An AMI establishes high-frequency two-way communication to enable the transmission of information between power utilities and the customers in a smart home. A typical AMI sys- 
tem includes smart meters, communications infrastructures (e.g., edge routers, aggregators, operational gateways, and a utility head-end), networks, and meter data management systems (MDMS). Among these components, the smart meters periodically record energy consumption data and establish connections for real-time power monitoring and billing purposes. The availability of dynamic electricity pricing information enables load control devices (e.g., smart thermostats) to modulate the electricity demand according to pre-defined user preferences. Recently, DLMS/COSEM has emerged as a standardized application protocol for communication between power utilities and customers [34], and powerline metering evolution can be applied for communication performance analysis. Figure 3 illustrates a typical AMI system model, including the smart meters, the network infrastructures (e.g., routers, gateways, and aggregators), and the MDMS. Note that ZigBee, RFID, Wi-Fi, WiMAX, fiber or cellular networks can be used for communication among home area networks, building area networks, and utility wide area networks (WANs), in which DERs are suitable for small-scale power generation. Both power lines and communication lines are established to achieve bidirectional energy and information interactions.

To obtain customers' actual power consumption patterns for energy load forecasting, AMI data are analyzed at various scales, ranging from very short-term load forecasting to long term load forecasting at the system level, regional level, feeder level, and consumer level [35]. Privacy-aware smart metering should also be considered for the purposes of metering for billing and metering for operations [36], for which sampling frequency, attribution and exactness are all relevant concerns.

\subsection{Supply-Demand Balance}

The issues related to energy management for supply-demand balance in a smart home are addressed through the concepts of demand response and vehicle-to-grid technology.

\subsubsection{Demand Response}

Demand response refers to reducing or shifting customers' normal power usage in response to periodic changes in electricity price and to providing incentive payments to induce lower power consumption in cases of high wholesale electricity market prices or power system reliability being jeopardized. Demand response allows energy customers to act as virtual power plants by voluntarily lowering their electricity demands to reduce electricity price volatility, mitigate the power of the generation market, and enhance power stability and reliability. Demand response mechanisms primarily include pricing-based demand response (e.g., time-of-use pricing, real-time pricing, and critical peak pricing) and incentive-based demand response (e.g., direct load control, interruptible load, demand-side bidding, and emergency demand response). Of these, incentive-based demand response typically involves providing customers with a flat retail electricity rate accompanied by more voluntary incentives to induce demand response, in contrast to real-time pricing or critical peak pricing schemes.

Cooperative demand response can be applied to realize efficient load management such that demand elasticity and consumer response enable load reduction. In a smart home scenario, non-coordinated responses from residential customers may cause peak rebounds during periods of lower prices; by contrast, coordinated demand response schemes can help to mitigate these peak rebounds. Such schemes can be formulated as a bi-level optimization problem (e.g., particle swarm optimization) and further transformed into equivalent single-level problems, which can then be solved by applying distributed iterative algorithms [37] in which home load management

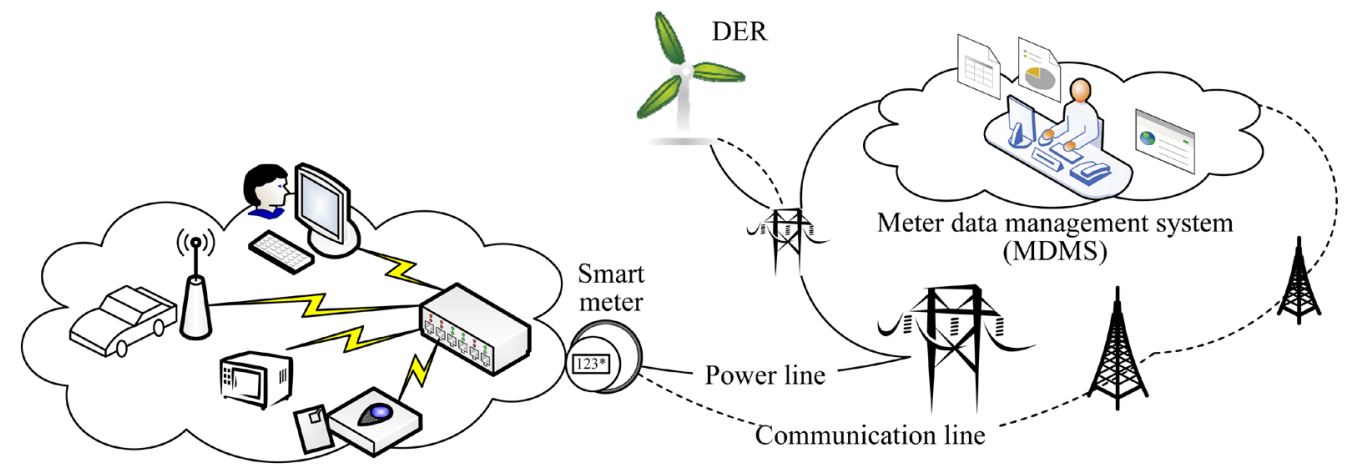

Figure 3. A typical AMI system model. 
(HLM) modules are embedded in smart meters as associated autonomous agents. Compared with noncooperative response strategies (i.e., Nash equilibria) for the oneshot demand management game, the Pareto-optimal response strategy is a popular approach to reducing electricity costs [38], and an incentive-compatible triggerand-punishment mechanism can be introduced in a demand response strategy to avoid the noncooperative behaviors of selfish consumers. Moreover, demand response strategies should be designed according to dynamic pricing tariffs, the seasons, and the weather, and uncertainties in demand response can be addressed as a stochastic unit commitment problem to enhance the reliability of the applied strategies.

\subsubsection{Vehicle-to-Grid}

Vehicle-to-grid (V2G) technology is an emerging network component of smart grids that involves battery-powered vehicles, local aggregators, and a remote central authority. In V2G networks, bidirectional power transmission and information communication are established to substantially enhance the power grid performance by alleviating peaks in power consumption. The vehicles perform both charging and discharging operations along with providing necessary information services for efficient power management and energy balance. The communications infrastructures between the vehicles and the power grid can facilitate better power supply-demand balance and consequently improve energy efficiency and reliability. In a smart home scenario, geographically scattered battery-powered vehicles play different roles [39] and, accordingly, have different responsibilities in their interactions with the power grid.

1) Energy demand: a vehicle acts as an energy consumer while accessing a local aggregator for power charging. This is the vehicle's traditional role, in which power flows from the power grid to the vehicle.

2) Energy storage: a vehicle acts as an energy store once it is fully charged. The fully charged vehicle becomes a distributed energy storage unit, which may potentially provide electricity to the power grid or other vehicles.

3) Energy supply: a vehicle can act as a small portable power plant that provides energy support by feeding its stored power back into the power grid. This discharging operation can relieve load peaks to achieve demand response balance. Note that both centralized and distributed discharging modes exist for such a vehicle. Of these, the centralized discharging mode refers to the mode in which a vehicle feeds its energy directly back into the power grid for centralized energy dispatch, whereas the distributed discharging mode refers to the mode in which a vehicle feeds its power to neighboring charging vehicles for local energy utilization. These two discharging modes provide a flexible energy supply to either the central power grid or charging vehicles in the local neighborhood, although the distributed discharging mode is more attractive for reducing operational costs and emission losses.

\section{Perspectives on IoT Energy Management}

IoT energy management exhibits several unique characteristics and perspectives involving the goals of green perception, green communication, and green computing.

\subsection{Supply Side}

The future IoT will realize the ubiquitous interconnections of things via cyber-physical-social spaces, and the relevant energy issues will possess the following characteristics.

\subsubsection{Space-Time Consistency}

Energy management considers both the spatial dimension (e.g., balance between energy supply and energy demand in different areas) and the temporal dimension (e.g., preservation of sufficient energy for future utilization) to achieve space-time consistency. However, IoT energy management will gradually eliminate the physical limitations of space and time. With such space and time constraints to overcome, IoT energy management can be performed at any time, in any place, and through any available methods.

\subsubsection{Heterogeneity}

Heterogeneity primarily refers to the heterogeneous devices, networks, and services that are closely associated with energy management. Heterogeneity promotes the ability to achieve a balance between energy consumption and energy efficiency and facilitates the development of a hybrid resource-optimized configuration. 


\subsubsection{Dynamic}

Dynamic topologies, services, and power supply schemes are encountered in wireless sensing and networking scenarios. Energy management should address the unstable and unpredictable nature of the power characteristics in these scenarios along with the utilization of renewable energy sources [40]. Meanwhile, energy prediction is a popular means of achieving efficient energy management and addressing potential future scenarios. Energy consumption and availability prediction approaches should be inspired by logical human thought and reasoning, and adaptive prediction models should be developed that can adapt to changes in energy consumption patterns.

\subsubsection{Social Attributes}

Social attributes play a vital role in the IoT, and a multi-attribute energy management model should be established that considers the ownerships and affiliation relationships of the relevant entities. The social responsibility of a power enterprise is primarily aimed at realizing a sustainable electricity system.

\subsubsection{Self-Managed Operation}

Self-management releases human beings from the responsibilities of energy management and enables intelligent energy-efficiency adaptations through the application of autonomous and autonomic systems in open, distributed networks. In a self-managing power system, energy resources are regarded as cognitive entities that are capable of self-management, including configuration, optimization, healing, and learning.

\subsection{The Convergence of Resource Collection, Communication and Computing}

Resource collection, communication and computing must be inseparably interconnected with each other to promote the green IoT, the purpose of which is to reduce the energy consumption of communication and computing infrastructures by adopting power-efficient mechanisms to achieve environmental sustainability. The convergence of resource collection, communication and computing has the potential to significantly reduce energy consumption and enhance energy efficiency. It influences IoT energy management in terms of connecting networked entities to establish green perception, communication, and computing.

\subsubsection{Green Perception}

For energy harvesting/storage, efficient sensing/processing, and energy buffering, standardized solutions are required for seamlessly integrating resource constrained devices into the networks. For instance, in typical wireless sensor and actuator networks (WSANs), the sensors and actuators are organized via large multihop topologies.

\subsubsection{Green Communication}

IP-based communication protocols are available for use in all IoT layers. For example, IPv6 over low-power wireless personal area networks (6LoWPAN) is suitable for the sensor-actuator layer, routing over low-power and lossy networks (ROLL) is appropriate for the network layer, and constrained RESTful environments (CoRE) can be used in the application layer. Both physical communication infrastructures and communication protocols have evolved to achieve the convergence of resource collection and computing.

\subsubsection{Green Computing}

The primary objective of green computing is to enable more energy-efficient use of resources along with efficiency improvement and energy conservation. In cloud computing, energy conservation has been extensively studied with regard to aspects such as VM migration and consolidation/allocation algorithms [41]. In particular, efficient green algorithms should consider the trade-offs of performance and cost that are encountered when applying power-saving policies in cloud computing.

\subsection{Internet of Energy}

The IoE is regarded as a web-enabled smart grid, or an Internet-based energy system, referring to the transformation of the traditional power grid into a smart grid. The smart grid represents a paradigm for enabling efficient energy generation, transmission, distribution, and consumption throughout the entire cycle of activity, for which small, lightweight, and resource-constrained devices with pervasive computing capabilities are advocated 
for use as key components in the deployment of a ubiquitous energy management scheme [42]. Following the trends of the green IoT, ICT technologies (e.g., transceivers, protocols, and services) should be optimized to address the inherent hardware limitations of the associated physical devices in the IoE context. An interactive mapping exits between the real deployment of energy management tools and virtual Internet components by coupling them via standard Internet-interoperable protocol stacks. The emergence of renewable energy sources and the growth of distributed generation have created new challenges regarding the use of the Internet as a communication tool for supporting energy management.

The IoE is expected to enable intelligent monitoring, control and regulation of electricity systems, with two major implications. For one, discrete and isolated energy systems will be able to establish interconnections through the Internet to provide support for and optimization of energy services. The communication infrastructures and energy infrastructures will be interconnected through information links and power links. For instance, the E-Energy project [43] was established by the Federal Ministry of Economics and Technology (BMWi) and the Federal Ministry for the Environment, Nature Conservation, Building and Nuclear Safety (BMU) to propel the development of the IoE through a task force on systems architecture, interoperability, legal frameworks, and market cultivation. Regarding the second major aspect of the IoE, efficient energy approaches are being developed to enhance the reliability, security, cost, performance, adaptability, migratability, compatibility, and scalability of energy management systems based on supply-side, demand-side, and supply-demand balance considerations.

\section{Conclusion}

In this work, we have established a taxonomy model for all IoT layers, including the sensor-actuator layer, network layer, and application layer. Considering the supply side, demand-side, and supply-demand balance aspects of IoT energy management, the associated energy issues were individually discussed. The sensor-actuator layer is responsible for energy harvesting and storage, efficient sensing and processing, and energy buffering for the achievement of green perception. The network layer focuses on energy distribution, efficient networking, and energy load balancing to achieve green communication. The application layer mainly addresses energy allocation, efficient infrastructure, energy metering and prediction for green computing. A case study of a smart home scenario was considered to provide an overview of the enabling technologies for energy management. Moreover, cyber-physical-social space fusion, the convergence of communication and computing, and the Internet of Energy were each discussed as new perspectives on IoT energy management.

\section{Acknowledgements}

This work was funded by the National Natural Science Foundation of China (61471035) and the Fundamental Research Funds for the Central Universities (06105031). In particular, it was supported by the Science and Technology Foundation of Beijing (Z141100002714003, Z151100002115041) and Cybermatics and Cyberspace International Science and Technology Cooperation Base.

\section{References}

[1] Huansheng, N. (2013) Unit and Ubiquitous Internet of Things. CRC Press, Taylor \& France Group, Boca Raton, London, New York.

[2] Gerards, M.E.T., Hurink, J.L. and Kuper, J. (2015) On the Interplay between Global DVFS and Scheduling Tasks with Precedence Constraints. IEEE Transactions on Computers, 64, 1742-1754.

[3] Okamura, H. and Dohi, T. (2015) Dynamic Power Management with Optimal Time-Out Policies. IEEE Systems Journal, PP, 1-11. http://dx.doi.org/10.1109/JSYST.2015.2450935

[4] Abbasi, Z., Pore, M., Banerjee, A. and Gupta, S.K.S. (2013) Multi-Tier Energy Buffering Management for IDCs with Heterogeneous Energy Storage Devices. 20th International Conference on High Performance Computing (HiPC), Bangalore, 18-21 December 2013, 368-377.

[5] Cao, Y., Jiang, T. and Zhang, Q. (2012) Reducing Electricity Cost of Smart Appliances via Energy Buffering Framework in Smart Grid. IEEE Transactions on Parallel and Distributed Systems, 23, 1572-1582. http://dx.doi.org/10.1109/TPDS.2012.126

[6] Padakandla, S., Prabuchandran, K.J. and Bhatnagar, S. (2015) Energy Sharing for Multiple Sensor Nodes with Finite Buffers. IEEE Transactions on Communications, 63, 1811-1823. http://dx.doi.org/10.1109/TCOMM.2015.2415777 
[7] Kim, J.-W., Barrado, J. and Jeon, D.-K. (2015) An Energy-Efficient Transmission Scheme for Real-Time Data in Wireless Sensor Networks. Sensors, 15, 11628-11652. http://www.mdpi.com/1424-8220/15/5/11628 http://dx.doi.org/10.3390/s150511628

[8] Xianjun, Z., Karady, G.G. and Ariaratnam, S.T. (2014) Optimal Allocation of CHP-Based Distributed Generation on Urban Energy Distribution Networks. IEEE Transactions on Sustainable Energy, 5, 246-253. http://dx.doi.org/10.1109/TSTE.2013.2278693

[9] Yu, W., Shiwen, M. and Nelms, R.M. (2014) Distributed Online Algorithm for Optimal Real-Time Energy Distribution in the Smart Grid. IEEE Internet of Things Journal, 1, 70-80. http://dx.doi.org/10.1109/JIOT.2014.2305667

[10] Nagarajan, A. and Ayyanar, R. (2015) Design and Strategy for the Deployment of Energy Storage Systems in a Distribution Feeder with Penetration of Renewable Resources. IEEE Transactions on Sustainable Energy, 6, 1085-1092. http://dx.doi.org/10.1109/TSTE.2014.2330294

[11] Jiang, H., Zhang, Y., Zhang, J.J., Gao, D.W. and Muljadi, E. (2015) Synchrophasor-Based Auxiliary Controller to Enhance the Voltage Stability of a Distribution System with High Renewable Energy Penetration. IEEE Transactions on Smart Grid, 6, 2107-2115. http://dx.doi.org/10.1109/TSG.2014.2387012

[12] Wei, D., Jin, Y., Vural, S., Moessner, K. and Tafazolli, R. (2011) An Energy-Efficient Clustering Solution for Wireless Sensor Networks. IEEE Transactions on Wireless Communications, 10, 3973-3983. http://dx.doi.org/10.1109/TWC.2011.092011.110717

[13] Luo, J., Rao, L. and Liu, X. (2015) Spatio-Temporal Load Balancing for Energy Cost Optimization in Distributed Internet Data Centers. IEEE Transactions on Cloud Computing, 3, 387-397. http://dx.doi.org/10.1109/TCC.2015.2415798

[14] Shao, H., Rao, L., Wang, Z., Liu, X., Wang, Z. and Ren, K. (2014) Optimal Load Balancing and Energy Cost Management for Internet Data Centers in Deregulated Electricity Markets. IEEE Transactions on Parallel and Distributed Systems, 25, 2659-2669. http://dx.doi.org/10.1109/TPDS.2013.227

[15] Paya, A. and Marinescu, D. (2015) Energy-Aware Load Balancing and Application Scaling for the Cloud Ecosystem. IEEE Transactions on Cloud Computing, PP, 1. http://dx.doi.org/10.1109/TCC.2015.2396059

[16] Nourian, M., Leong, A.S. and Dey, S. (2014) Optimal Energy Allocation for Kalman Filtering over Packet Dropping Links with Imperfect Acknowledgments and Energy Harvesting Constraints. IEEE Transactions on Automatic Control, 59, 2128-2143. http://dx.doi.org/10.1109/TAC.2014.2319011

[17] Liu, D., Chen, Y., Chai, K.K., Zhang, T. and Han, K. (2015) Joint User Association and Green Energy Allocation in HetNets with Hybrid Energy Sources. IEEE Wireless Communications and Networking Conference (WCNC), New Orleans, 9-12 March 2015, 1542-1547.

[18] Zhang, C., Wu, W., Huang, H. and Yu, H. (2012) Fair Energy Resource Allocation by Minority Game Algorithm for Smart Buildings. Design, Automation \& Test in Europe Conference \& Exhibition (DATE), Dresden, 12-16 March 2012, 63-68.

[19] Khalifa, T., Naik, K. and Nayak, A. (2011) A Survey of Communication Protocols for Automatic Meter Reading Applications. IEEE Communications Surveys \& Tutorials, 13, 168-182. http://dx.doi.org/10.1109/SURV.2011.041110.00058

[20] Donnal, J.S. and Leeb, S.B. (2015) Noncontact Power Meter. IEEE Sensors Journal, 15, 1161-1169. http://dx.doi.org/10.1109/JSEN.2014.2359619

[21] Jiang, R., Lu, R., Wang, Y., Luo, J., Shen, C. and Shen, X.S. (2014) Energy-Theft Detection Issues for Advanced Metering Infrastructure in Smart Grid. Tsinghua Science and Technology, 19, 105-120. http://dx.doi.org/10.1109/TST.2014.6787363

[22] Li, Y., Jia, Z. and Xie, S. (2014) Energy-Prediction Scheduler for Reconfigurable Systems in Energy-Harvesting Environment. IET Wireless Sensor Systems, 4, 80-85. http://dx.doi.org/10.1049/iet-wss.2012.0129

[23] Feng, T., Yang, L., Gu, Q., Hu, Y., Yan, T. and Yan, B. (2015) A Supervisory Control Strategy for Plug-In Hybrid Electric Vehicles Based on Energy Demand Prediction and Route Preview. IEEE Transactions on Vehicular Technology, 64, 1691-1700. http://dx.doi.org/10.1109/TVT.2014.2336378

[24] Hou, C. and Zhao, Q.C. (2015) Bayesian Prediction-Based Energy-Saving Algorithm for Embedded Intelligent Terminal. IEEE Transactions on Very Large Scale Integration (VLSI) Systems, 23, 2902-2912. http://dx.doi.org/10.1109/TVLSI.2014.2385791

[25] Deng, W., Liu, F., Jin, H., Li, B. and Li, D. (2014) Harnessing Renewable Energy in Cloud Datacenters: Opportunities and Challenges. IEEE Network, 28, 48-55. http://dx.doi.org/10.1109/MNET.2014.6724106

[26] Sun, D., Ge, B., Liang, W., Abu-Rub, H. and Peng, F.Z. (2015) An Energy Stored Quasi-Z-Source Cascade Multilevel Inverter-Based Photovoltaic Power Generation System. IEEE Transactions on Industrial Electronics, 62, 5458-5467. 
http://dx.doi.org/10.1109/TIE.2015.2407853

[27] Sundareswaran, K., Sankar, P., Nayak, P.S.R., Simon, S.P. and Palani, S. (2015) Enhanced Energy Output from a PV System under Partial Shaded Conditions through Artificial Bee Colony. IEEE Transactions on Sustainable Energy, 6, 198-209. http://dx.doi.org/10.1109/TSTE.2014.2363521

[28] Xu, W., Mu, C. and Jin, J. (2014) Novel Linear Iteration Maximum Power Point Tracking Algorithm for Photovoltaic Power Generation. IEEE Transactions on Applied Superconductivity, 24, 1-6. http://dx.doi.org/10.1109/tasc.2014.2333534

[29] Senanayaka, J.S.L. and Kolhe, M.L. (2015) Integration of Distributed Energy Systems in Micro-Grid Architecture for Making a Virtual Power Plant. 2015 IEEE International Conference on Signal Processing, Informatics, Communication and Energy Systems (SPICES), Kozhikode, 19-21 February 2015, 1-5. http://dx.doi.org/10.1109/SPICES.2015.7091500

[30] Anvari-Moghaddam, A., Monsef, H. and Rahimi-Kian, A. (2015) Optimal Smart Home Energy Management Considering Energy Saving and a Comfortable Lifestyle. IEEE Transactions on Smart Grid, 6, 324-332. http://dx.doi.org/10.1109/TSG.2014.2349352

[31] ONF Software-Defined Networking: The New Norm for Networks. White Paper. https://www.opennetworking.org

[32] Sezer, S., Scott-Hayward, S., Chouhan, P.K., Fraser, B., Lake, D., Finnegan, J., Viljoen, N., Miller, M. and Rao, N. (2013) Are We Ready for SDN? Implementation Challenges for Software-Defined Networks. IEEE Communications Magazine, 51, 36-43. http://dx.doi.org/10.1109/MCOM.2013.6553676

[33] Li, D., Shang, Y. and Chen, C. (2014) Software Defined Green Data Center Network with Exclusive Routing. 2014 Proceedings IEEE INFOCOM, Toronto, 27 April-2 May 2014, 1743-1751.

[34] Matanza, J., Alexandres, S. and Rodríguez-Morcillo, C. (2014) Advanced Metering Infrastructure Performance Using European Low-Voltage Power Line Communication Networks. IET Communications, 8, 1041-1047. http://dx.doi.org/10.1049/iet-com.2013.0793

[35] Quilumba, F.L., Lee, W.J., Huang, H., Wang, D.Y. and Szabados, R.L. (2015) Using Smart Meter Data to Improve the Accuracy of Intraday Load Forecasting Considering Customer Behavior Similarities. IEEE Transactions on Smart Grid, 6, 911-918. http://dx.doi.org/10.1109/TSG.2014.2364233

[36] Finster, S. and Baumgart, I. (2014) Privacy-Aware Smart Metering: A Survey. IEEE Communications Surveys \& Tutorials, 16, 1732-1745. http://dx.doi.org/10.1109/SURV.2014.052914.00090

[37] Safdarian, A., Fotuhi-Firuzabad, M. and Lehtonen, M. (2014) A Distributed Algorithm for Managing Residential Demand Response in Smart Grids. IEEE Transactions on Industrial Informatics, 10, 2385-2393. http://dx.doi.org/10.1109/TII.2014.2316639

[38] Ma, K., Hu, G. and Spanos, C.J. (2015) A Cooperative Demand Response Scheme Using Punishment Mechanism and Application to Industrial Refrigerated Warehouses. IEEE Transactions on Industrial Informatics, 11, 1520-1531. http://dx.doi.org/10.1109/TII.2015.2431219

[39] Liu, H., Ning, H., Zhang, Y., Xiong, Q. and Yang, L.T. (2014) Role-Dependent Privacy Preservation for Secure V2G Networks in the Smart Grid. IEEE Transactions on Information Forensics and Security, 9, 208-220. http://dx.doi.org/10.1109/TIFS.2013.2295032

[40] Choi, H.-S. and Rhee, W.-S. (2014) IoT-Based User-Driven Service Modeling Environment for a Smart Space Management System. Sensors, 14, 22039-22064. http://www.mdpi.com/1424-8220/14/11/22039 http://dx.doi.org/10.3390/s141122039

[41] Chiang, Y.J., Ouyang, Y.C. and Hsu, C.H. (2015) An Efficient Green Control Algorithm in Cloud Computing for Cost Optimization. IEEE Transactions on Cloud Computing, 3, 145-155. http://dx.doi.org/10.1109/TCC.2014.2350492

[42] Bui, N., Castellani, A.P., Casari, P. and Zorzi, M. (2012) The Internet of Energy: A Web-Enabled Smart Grid System. IEEE Network, 26, 39-45. http://dx.doi.org/10.1109/MNET.2012.6246751

[43] ONF E-Energy - Smart Grids Made in Germany. http://www.bmwi.de/English/Redaktion/Pdf/smart-energy-made-in-germany 